\title{
A note on the Penrose junction conditions
}

\author{
Michael Kunzinger * \\ Department of Mathematics, University of Vienna, Strudlhofg. 4 \\ A-1090 Wien, Austria \\ Roland Steinbauer ${ }^{\dagger}$ \\ Department of Mathematics, University of Vienna, Strudlhofg. 4 \\ Institute for Theoretical Physics, University of Vienna, Boltzmanng. 5 \\ A-1090 Wien, Austria
}

\begin{abstract}
Impulsive pp-waves are commonly described either by a distributional spacetime metric or, alternatively, by a continuous one. The transformation $T$ relating these forms clearly has to be discontinuous, which causes two basic problems: First, it changes the manifold structure and second, the pullback of the distributional form of the metric under $T$ is not well defined within classical distribution theory. Nevertheless, from a physical point of view both pictures are equivalent. In this work, after calculating $T$ as well as the "Rosen"-form of the metric in the general case of a pp-wave with arbitrary wave profile we give a precise meaning to the term "physicially equivalent" by interpreting $T$ as the distributional limit of a suitably regularized sequence of diffeomorphisms. Moreover, it is shown that $T$ provides an example of a generalized coordinate transformation in the sense of Colombeau's generalized functions.
\end{abstract}

Keywords: Impulsive pp-waves, distributional metric, discontinuous coordinate transformation, Colombeau generalized functions. PACS-numbers: 04.20.-q, 04.20.Cv, 04.30.-w, 02.30.Sa, 02.90.+p. $M S C:$ 83Cxx, 46F10, 83C $35,35 \mathrm{Dxx}$

Report no: UWThPh - $1998-57$

\section{INTRODUCTION}

Plane fronted gravitational waves with parallel rays (pp-waves) are spacetimes characterized by the existence of a covariantly constant null vector field, which allows to write the metric in the form [1]

$$
d s^{2}=H d u^{2}+2 d \zeta d \bar{\zeta}-2 d u d v,
$$

where $H(u, \zeta, \bar{\zeta})$ is a function of the the retarded time coordinate $u$ and the transverse complex coordinates $\zeta$ and $\bar{\zeta}$. Impulsive pp-waves may now be defined by setting [2]

$$
H(u, \zeta, \bar{\zeta})=f(\zeta, \bar{\zeta}) \delta(u)
$$

where $f$ denotes a smooth function of $\zeta, \bar{\zeta}$ and $\delta$ is the Dirac- $\delta$-distribution. Such spacetimes arise in a very natural manner as the ultrarelativistic limit of Kerr-Newman black holes [3-5] and multipole solutions of the Weyl family as recently shown by Podolsky and Griffiths [6]. Moreover these geometries play an important role in the description of scattering processes at the Planck scale $[7,8]$.

While the form (1) of the metric very clearly demonstrates the nature of the impulsive wave, i.e. that the spacetime is flat everywhere except for the null hyperplane $u=0$ where the $\delta$-like shock is located, it has the obvious disadvantage of involving distributional coefficients. However, due to the simple form of the metric the curvature tensor can be calculated and is itself proportional to the $\delta$-distribution. Moreover, the geometry of impulsive pp-waves was described recently $[9,10]$ by the authors entirely in the distributional picture. The key idea was to use a careful regularization procedure which allowed to handle the nonlinear singular geodesic and geodesic deviation equations in a mathematically satisfactory way.

On the other hand, impulsive pp-waves are frequently described by a different spacetime metric which is actually continuous (see $[2,11,12]$ and, for the general case, [13]). In the special case of an impulsive plane (linearly polarized) wave, i.e. $f=1 / 2\left(\zeta^{2}+\bar{\zeta}^{2}\right)$, it takes the form

*Electronic mail: Michael.Kunzinger@univie.ac.at

${ }^{\dagger}$ Electronic mail: roland.steinbauer@univie.ac.at 


$$
d s^{2}=\left(1+u_{+}\right)^{2} d X^{2}+\left(1-u_{+}\right)^{2} d Y^{2}-2 d u d V .
$$

where $u_{+}$denotes the "kink" function $u_{+}=\theta(u) u$. This form of the metric has the advantage that only the curvature tensor involves distributions while the metric can be treated "classically." Moreover, if one constructs impulsive pp-waves by glueing together two copies of Minkowski space along the hypersurface $u=0$ with a coordinate shift (according to Penrose's scissors and paste approach [2]) a theorem of Clarke and Dray [14] ensures the existence of a $C^{1}$-atlas in which the metric components are continuous.

Clearly a transformation relating the metrics (1) and (3) cannot even be continuous, hence strictly speaking it changes (even the topological structure of) the manifold. In the case of a plane wave this discontinuous change of variables is given by the so called Penrose junction conditions [2] (for the general vacuum case again see [11])

$$
\begin{aligned}
\sqrt{2} \zeta & =X\left(1+u_{+}\right)+i Y\left(1-u_{+}\right) \\
v & =V+\frac{1}{2} X^{2}\left(u_{+}+\theta(u)\right)+\frac{1}{2} Y^{2}\left(u_{+}-\theta(u)\right) \\
u & =u
\end{aligned}
$$

However, the two (mathematically) distinct spacetimes are equivalent from a physical point of view, i.e. the geodesics and the particle motion agree [15]. More precisely, if one computes the geodesics for the continuous metric and then (formally) applies the discontinuous transformation one obtains exactly the geodesics as derived in the distributional picture in [9].

Nevertheless, it has to be noted that the actual calculation of the continuous metric (3) from the distributional form (1) as well as the transformation of the geodesics involve undefined nonlinear operations with distributions which are dealt with by using ad hoc multiplication rules; in this case $\theta^{2}=\theta, \theta u_{+}=u_{+}, u_{+} \delta=0=u_{+}^{2} \delta$ and $\theta \delta=1 / 2 \delta$. Such a seemingly harmless operational approach to introducing nonlinearities into linear distribution theory may work out in certain cases but in just as many instances it will lead seriously astray. Using the above, i.e. exactly the "rules" appearing in the situation under consideration we get $(\theta \theta) \delta=\theta \delta=1 / 2 \delta \neq 1 / 4 \delta=\theta(\theta \delta)$. For examples of the difficulties that may result from such manipualtions both in mathematics and in physics, cf. [16] and [17]. Especially in the context of nonlinear differential equations involving generalized functions (in this case: the geodesic equations) it turns out that the method of choice for determining which multiplications work out and which don't is to first regularize the singularities, then carry out the nonlinear operations and, finally, compute the distributional limits, if they exist (cf. [18], p. 180).

By following this route, in this paper we give a precise meaning to the above-mentioned "physical equivalence," interpreting the discontinuous change of variables as the (distributional) limit of a family of smoothened transformations. The key fact is to note that the coordinate lines in the new variables defined in (4) are exactly the geodesics of the metric (1) with vanishing initial speed in the $\zeta-, \bar{\zeta}-$ and $v$-directions.

More precisely, we start with the distributional form (1) of the metric, which we turn into a sandwich wave by regularizing the $\delta$ distribution in a completely general manner. Then we carry out a transformation analogous to (4) and calculate the distributional limit of the metric to arrive at the continuous form, which in the special case considered above precisely agrees with (3). In calculating this limit, which is totally independent of the form of the regularization (hence "natural"), we make use of the results in [9] and [10]. In the sandwich wave - picture both forms of the impulsive wave, i.e. the metrics (1) and (3), arise as (distributional) limits in different coordinate systems (see also the remarks in Sec. V).

\section{THE SMOOTHENED TRANSFORMATION}

Since our considerations depend heavily upon the results of $[9,10]$ we shall stick to the real coordinates used there and start with an impulsive pp-metric of the form

$$
d s^{2}=f\left(x^{i}\right) \delta(u) d u^{2}-d u d v+\sum_{i=1}^{2}\left(d x^{i}\right)^{2},
$$

where $x^{i}=(x, y)$ denotes the transverse coordinates spanning the wave surface. The first step is now to regularize the $\delta$-distribution by a sequence of (smooth) functions $\rho_{\varepsilon}(0<\varepsilon \leq 1)$. Since we are interested in the most general result we will only assume the following minimal set of conditions on the regularization (so called generalized $\delta$-function)

(a) $\operatorname{supp}\left(\rho_{\varepsilon}\right) \subseteq[-\varepsilon, \varepsilon]$, 


$$
\begin{aligned}
& \text { (b) } \int \rho_{\varepsilon}(x) d x \rightarrow 1 \quad(\varepsilon \rightarrow 0) \text { and } \\
& \text { (c) } \int\left|\rho_{\varepsilon}(x)\right| d x \leq C \text { for small } \varepsilon .
\end{aligned}
$$

Note that condition (a) is choosen in order to avoid technicalities in the calculation of the distributional limits which, however, remain valid if (a) is replaced by

$$
\text { (a') } \operatorname{supp}\left(\rho_{\varepsilon}\right) \rightarrow\{0\} \quad(\varepsilon \rightarrow 0) .
$$

Hence we are left with the following sequence of sandwich waves

$$
d s_{\varepsilon}^{2}=f\left(x^{i}\right) \rho_{\varepsilon}(u) d u^{2}-d u d v+\sum_{i=1}^{2}\left(d x^{i}\right)^{2} .
$$

The corresponding geodesic equations are given by

$$
\begin{aligned}
\ddot{x}_{\varepsilon}^{i}(u) & =\frac{1}{2} \partial_{i} f\left(x_{\varepsilon}^{j}(u)\right) \rho_{\varepsilon}(u) \\
\ddot{v}_{\varepsilon}(u) & =f\left(x_{\varepsilon}^{j}(u)\right) \dot{\rho}_{\varepsilon}(u)+2 \partial_{i} f\left(x_{\varepsilon}^{j}(u)\right) \dot{x}_{\varepsilon}^{i}(u) \rho_{\varepsilon}(u),
\end{aligned}
$$

where we have used $u$ as an affine parameter. Since we are only interested in geodesics with the special initial conditions $x_{\varepsilon}^{i}(-1)=x_{0}^{i}, v_{\varepsilon}(-1)=v_{0}$ and $\dot{x}_{\varepsilon}^{i}(-1)=0=\dot{v}_{\varepsilon}(-1)$, we adopt the notation $x_{\varepsilon}^{i}\left(x_{0}^{i}, u\right)$ and $v_{\varepsilon}\left(v_{0}, x_{0}^{i}, u\right)$ for these geodesics with vanishing initial speed. They obey the following (implicit) set of equations

$$
\begin{aligned}
x_{\varepsilon}^{i}\left(x_{0}^{i}, u\right) & =x_{0}^{i}+\frac{1}{2} \int_{-\varepsilon}^{u} \int_{-\varepsilon}^{s} \partial_{i} f\left(x_{\varepsilon}^{j}\left(x_{0}^{k}, r\right)\right) \rho_{\varepsilon}(r) d r d s \\
v_{\varepsilon}\left(v_{0}, x_{0}^{i}, u\right) & \left.=v_{0}+\int_{-\varepsilon}^{u} f\left(x_{\varepsilon}^{j}\left(x_{0}^{k}, s\right)\right) \rho_{\varepsilon}(s) d s+\int_{-\varepsilon}^{u} \int_{-\varepsilon}^{s} \partial_{i} f\left(x_{\varepsilon}^{j}\left(x_{0}^{k}, r\right)\right) \dot{x}_{\varepsilon}^{i}\left(x_{0}^{k}, r\right)\right) \rho_{\varepsilon}(r) d r d s
\end{aligned}
$$

In [10] it was shown that these equations are uniquely solvable (even) within the framework of Colombeau's algebras of generalized functions [18-20], which provide a suitable setting for singular differential equations. Moreover the unique solution possesses the following distributional limit, independent of the concrete shape of the regularization

$$
\begin{aligned}
x_{\varepsilon}^{i}\left(x_{0}^{j}, u\right) & \rightarrow x_{0}^{i}+\frac{1}{2} \partial_{i} f\left(x_{0}^{j}\right) u_{+} \\
v_{\varepsilon}\left(v_{0}, x_{0}^{j}, u\right) & \rightarrow v_{0}+f\left(x_{0}^{j}\right) \theta(u)+\frac{1}{4} \partial_{i} f\left(x_{0}^{j}\right) \partial^{i} f\left(x_{0}^{j}\right) u_{+} \cdot
\end{aligned}
$$

Let us now consider the transformation $T_{\varepsilon}:\left(u, v, x^{i}\right) \rightarrow\left(u, V, X^{i}\right)$ (where $\left.X^{i}=(X, Y)\right)$ given implicitly by $(10)$ and depending on the regularization parameter $\varepsilon$ according to

$$
\begin{aligned}
x^{i} & =x_{\varepsilon}^{i}\left(X^{j}, u\right) \\
v & =v_{\varepsilon}\left(V, X^{j}, u\right)
\end{aligned}
$$

which is precisely analogous (and in the limit $\varepsilon \rightarrow 0$ and the special case of a plane wave actually reduces) to (4). We claim that $T_{\varepsilon}$ yields a coordinate transformation such that the new coordinates $(u, V, X, Y)$ are constant along the geodesics given by (10). While the latter property follows directly from the construction, we still have to verify that $T_{\varepsilon}$ is a diffeomorphism in a spacetime region containing the shock-hypersurface $u=0$. To establish this property we employ a global univalence theorem by Gale and Nikaido ( $[21]$, Thm. 4) stating that any differentiable $F: \Omega \rightarrow \mathbb{R}^{n}$, where $\Omega$ is a closed rectangular region in $\mathbb{R}^{n}$ is univalent (injective) if all principal minors of its Jacobian $J(x)$ are positive. Since

$$
\frac{\partial\left(u, x^{1}, x^{2}, v\right)}{\partial\left(u, X^{1}, X^{2}, V\right)}=\left|\begin{array}{cccc}
1 & 0 & 0 & 0 \\
\frac{\partial x_{\varepsilon}^{1}}{\partial u} & \frac{\partial x_{\varepsilon}^{1}}{\partial X^{1}} & \frac{\partial x_{\varepsilon}^{1}}{\partial X^{2}} & 0 \\
\frac{\partial x_{\varepsilon}^{2}}{\partial u} & \frac{\partial x_{\varepsilon}^{2}}{\partial X^{1}} & \frac{\partial x_{\varepsilon}^{2}}{\partial X^{2}} & 0 \\
\frac{\partial v_{\varepsilon}}{\partial u} & \frac{\partial v_{\varepsilon}}{\partial X^{1}} & \frac{\partial v_{\varepsilon}}{\partial X^{2}} & 1
\end{array}\right|
$$


we have to find estimates for

$$
\frac{\partial x_{\varepsilon}^{i}}{\partial X^{j}}=\delta_{j}^{i}+\frac{1}{2} \int_{-\varepsilon}^{u} \int_{-\varepsilon}^{s}\left(\partial_{m} \partial_{i} f\right)\left(x_{\varepsilon}^{k}\left(X^{l}, r\right)\right) \frac{\partial x_{\varepsilon}^{m}}{\partial X^{j}}\left(X^{l}, r\right) \rho_{\varepsilon}(r) d r d s .
$$

If $X^{l}$ varies in a compact region $K$ of $\mathbb{R}^{2}$ and $-1 \leq p \leq u<\infty$ it follows from a straightforward modification of the appendix of [9] that for small $\varepsilon$ the terms $x_{\varepsilon}^{k}\left(X^{l}, p\right)$ remain bounded, independently of $X^{l}, p$ and $\varepsilon$. Let $g(u):=\sup \left\{\sum_{i=1}^{2}\left|\frac{\partial x_{\varepsilon}^{i}}{\partial X^{j}}\left(X^{k}, p\right)\right|: X^{k} \in K,-1 \leq p \leq u\right\}$. Then (14) gives

$$
|g(u)| \leq C_{1}+C_{2} \int_{-\varepsilon}^{u}|g(s)| d s
$$

so Gronwall's lemma implies the same boundedness property for $\frac{\partial x_{\varepsilon}^{i}}{\partial X^{j}}$. Using these estimates it follows from (14) that for small $\varepsilon$ and for small $u>0$ (depending on $\left\|\partial_{i} \partial_{j} f\right\|_{\infty}$ in a compact region and on $C$ from (6c)) $\frac{\partial x_{\varepsilon}^{i}}{\partial X^{j}}$ will remain arbitrarily small (for $i \neq j$ ) or arbitrarily close to 1 (for $i=j$ ), respectively. Hence all principal minors of (13) are indeed positive in a suitable rectangular region (independent of $\epsilon$ ) containing the shock hypersurface $u=0$ which establishes our claim. We note that this result in particular implies that for small $\varepsilon$ the geodesics (10) do not cross in this region.

In the new coordinates $(u, V, X, Y)$ the metric tensor takes the form

$$
d s_{\varepsilon}^{2}=-d u d V+\left(2 \sum_{i=1}^{2}\left(\dot{x}_{\varepsilon}^{i} \partial_{j} x_{\varepsilon}^{i}\right)-\partial_{j} v_{\varepsilon}\right) d u d X^{j}+\sum_{i=1}^{2}\left(\partial_{j} x_{\varepsilon}^{i} d X^{j}\right)^{2},
$$

where ${ }^{\cdot}$ and $\partial_{i}$ denote derivatives with respect to $u$ and $X^{i}$ respectively and we have omitted the straightforward computation showing that $g_{u u}=0$.

\section{DISTRIBUTIONAL LIMIT}

Our next task is to compute the distributional limit of the metric (15) as $\varepsilon \rightarrow 0$. To reduce the notational complexity we are going to suppress the explicit dependence of test functions on the variables $X^{i}$ and $V$, i.e. we will write $\varphi(u)$ for $\varphi \in \mathcal{D}\left(\mathbb{R}^{4}\right)$ and will simply drop the integrations with respect to $X^{i}$ and $V$. This abuse of notation is admissible due to the uniform boundedness properties (in $X^{i}$ and $V$ ) established above and serves to reduce the number of integrals in the sequel by 3 . We start out with the coefficient

$$
\begin{aligned}
g_{u X}= & \sum_{i=1}^{2}\left(\int_{-\varepsilon}^{u} \partial_{i} f\left(x_{\varepsilon}^{j}\left(X^{k}, s\right)\right) \rho_{\varepsilon}(s) d s \partial_{1}\left(X^{i}+\frac{1}{2} \int_{-\varepsilon}^{u} \int_{-\varepsilon}^{s} \partial_{i} f\left(x_{\varepsilon}^{j}\left(X^{k}, r\right) \rho_{\varepsilon}(r) d r d s\right)\right)\right. \\
& -\partial_{1}\left(V+\int_{-\varepsilon}^{u} f\left(x_{\varepsilon}^{j}\left(X^{k}, r\right)\right) \rho_{\varepsilon} d r+\int_{-\varepsilon}^{u} \int_{-\varepsilon}^{s} \partial_{l} f\left(x_{\varepsilon}^{j}\left(X^{k}, r\right) \dot{x}_{\varepsilon}^{l}\left(X^{j}, r\right) \rho_{\varepsilon}(r) d r d s\right)\right.
\end{aligned}
$$

The first term of this expression can be written as

$$
\sum_{i=1}^{2}(\delta_{i}^{1} \underbrace{\int_{-\varepsilon}^{u} \partial_{i} f\left(x_{\varepsilon}^{j}\left(X^{k}, s\right)\right) \rho_{\varepsilon}(r) d r}_{\rightarrow \partial_{i} f\left(X^{j}\right) \theta}+\underbrace{\frac{1}{2} \int_{-\varepsilon}^{u} \partial_{i} f\left(x_{\varepsilon}^{j}\left(X^{k}, r\right)\right) \rho_{\varepsilon}(r) d r \partial_{1} \int_{-\varepsilon}^{u} \int_{-\varepsilon}^{s} \partial_{i} f\left(x_{\varepsilon}^{j}\left(X^{k}, r\right) \rho_{\varepsilon}(r) d r d s\right)}_{=: A}
$$

For any test function $\varphi$ we have

$$
\langle 2 A, \varphi\rangle=\int_{-\varepsilon}^{\infty} \varphi(u) \int_{-\varepsilon}^{u} \partial_{i} f\left(x_{\varepsilon}^{j}\left(X^{k}, r\right)\right) \rho_{\varepsilon}(r) d r \partial_{1} \int_{-\varepsilon}^{u} \int_{-\varepsilon}^{s} \partial_{i} f\left(x_{\varepsilon}^{j}\left(X^{k}, r\right) \rho_{\varepsilon}(r) d r d s d u\right.
$$


Splitting this integral into a sum of the form

$$
\int_{-\varepsilon}^{\varepsilon} \ldots \int_{-\varepsilon}^{u} \ldots \int_{-\varepsilon}^{u} \int_{-\varepsilon}^{s} \ldots+\int_{\varepsilon}^{\infty} \ldots \int_{-\varepsilon}^{u} \ldots \int_{-\varepsilon}^{\varepsilon} \int_{-\varepsilon}^{s} \ldots+\int_{\varepsilon}^{\infty} \ldots \int_{-\varepsilon}^{u} \ldots \int_{\varepsilon}^{u} \int_{-\varepsilon}^{s} \ldots
$$

the boundedness arguments following (14) imply that the first two summands converge to 0. Also, by (11) in [9] and by (10) we obtain

$$
\lim _{\varepsilon \rightarrow 0} \sup _{|r|<\varepsilon}\left|\partial_{i}\left(f\left(x_{\varepsilon}^{j}\left(X^{k}, r\right)\right)\right)-\partial_{i} f\left(X^{k}\right)\right|=0
$$

uniformly for $X^{k}$ in compact sets. For later use we note that by the same reasoning also

$$
\lim _{\varepsilon \rightarrow 0} \sup _{|r|<\varepsilon}\left|\partial_{i}\left(\partial_{j} f\left(x_{\varepsilon}^{j}\left(X^{k}, r\right)\right)\right)-\partial_{i j} f\left(X^{k}\right)\right|=0
$$

uniformly for $X^{k}$ in compact sets. Hence by a direct estimation the limit of the remaining term is $\left\langle\partial_{i} f\left(X^{j}\right) \partial_{1} \partial_{i} f\left(X^{j}\right) u_{+}, \varphi\right\rangle$, so the distributional limit of the first term in (16) is $\partial_{1} f\left(X^{j}\right) \theta+\frac{1}{2} \sum_{i=1}^{2} \partial_{i} f\left(X^{j}\right) \partial_{i} \partial_{1} f\left(X^{j}\right) u_{+}$. Turning now to the second term in (16) we obtain from (18) and (6b):

$$
\partial_{1} \int_{-\varepsilon}^{u} f\left(x_{\varepsilon}^{j}\left(X^{k}, r\right)\right) \rho_{\varepsilon}(r) d r \rightarrow \partial_{1} f\left(X^{j}\right) \theta
$$

Inserting (10) it remains to calculate the distributional limit of

$$
\begin{aligned}
& \frac{1}{2} \int_{-\varepsilon}^{u} \int_{-\varepsilon}^{s} \partial_{1}\left(\partial _ { i } f ( x _ { \varepsilon } ^ { j } ( X ^ { k } , r ) ) \rho _ { \varepsilon } ( r ) \int _ { - \varepsilon } ^ { r } \partial _ { i } f \left(x_{\varepsilon}^{j}\left(X^{k}, q\right) \rho_{\varepsilon}(q) d q d r d s\right.\right. \\
& +\frac{1}{2} \int_{-\varepsilon}^{u} \int_{-\varepsilon}^{s} \partial_{i} f\left(x _ { \varepsilon } ^ { j } ( X ^ { k } , r ) \rho _ { \varepsilon } ( r ) \int _ { - \varepsilon } ^ { r } \partial _ { 1 } \left(\partial_{i} f\left(x_{\varepsilon}^{j}\left(X^{k}, q\right)\right) \rho_{\varepsilon}(q) d q d r d s .\right.\right.
\end{aligned}
$$

A splitting scheme as in (17) for both these terms, (18) and the fact that

$$
\int_{\varepsilon}^{u} \int_{-\varepsilon}^{s} \rho_{\varepsilon}(r) \int_{-\varepsilon}^{r} \rho_{\varepsilon}(q) d q d r d s \rightarrow \frac{1}{2} u_{+}
$$

imply that each summand in (20) converges distributionally to $\frac{1}{4} \partial_{i} f\left(X^{j}\right) \partial_{1 i} f\left(X^{j}\right) u_{+}$.

An analogous argument holds for $g_{u Y}$. Summing up, we obtain

$$
g_{u X} \stackrel{\mathcal{D}^{\prime}}{\longrightarrow} 0 \quad \text { and } \quad g_{u Y} \stackrel{\mathcal{D}^{\prime}}{\longrightarrow} 0 \quad \text { for } \varepsilon \rightarrow 0 .
$$

If we write

$$
g_{X X}=\sum_{i=1}^{2}\left(\partial_{1}\left(X^{i}+\frac{1}{2} \int_{-\varepsilon}^{u} \int_{-\varepsilon}^{s} \partial_{i} f\left(x_{\varepsilon}^{j}\left(X^{k}, r\right)\right) \rho_{\varepsilon}(r) d r d s\right)\right)^{2}=: \sum_{i=1}^{2} R_{i}
$$

then

$$
R_{i}=\delta_{i}^{1}+\underbrace{\int_{-\varepsilon}^{u} \int_{-\varepsilon}^{s} \partial_{1}\left(\partial_{1} f\left(x_{\varepsilon}^{j}\left(X^{k}, r\right)\right)\right) \rho_{\varepsilon}(r) d r d s}_{\rightarrow \partial_{11} f\left(X^{j}\right) u_{+}}+\underbrace{\frac{1}{4}\left(\int_{-\varepsilon}^{u} \int_{-\varepsilon}^{s} \partial_{1}\left(\partial_{i} f\left(x_{\varepsilon}^{j}\left(X^{k}, r\right)\right)\right) \rho_{\varepsilon}(r) d r d s\right)^{2}}_{=: B}
$$


and by a similar argument as above it follows that the distributional limit of the derivative $\partial_{u} B$ is $\frac{1}{2}\left(\partial_{1} \partial_{i} f\left(X^{j}\right)\right)^{2} u_{+}$. Since taking primitives (i.e. convoluting with $\theta$ ) is separately continuous on the convolution algebra of distributions supported in an acute cone and since taking tensor products of distributions is separately continuous as well we get

$$
R_{i} \rightarrow \delta_{i}^{1}+\partial_{11} f\left(X^{j}\right) u_{+}+\frac{1}{2}\left(\partial_{1} \partial_{i} f\left(X^{j}\right)\right)^{2} \frac{u_{+}^{2}}{2} .
$$

Thus

$$
\begin{aligned}
& g_{X X} \stackrel{\mathcal{D}^{\prime}}{\longrightarrow}\left(1+\frac{1}{2} \partial_{11} f\left(X^{j}\right) u_{+}\right)^{2}+\frac{1}{4}\left(\partial_{12} f\left(X^{j}\right)\right)^{2} u_{+}^{2} \\
& g_{Y Y} \stackrel{\mathcal{D}^{\prime}}{\longrightarrow}\left(1+\frac{1}{2} \partial_{22} f\left(X^{j}\right) u_{+}\right)^{2}+\frac{1}{4}\left(\partial_{12} f\left(X^{j}\right)\right)^{2} u_{+}^{2}
\end{aligned}
$$

Finally, we turn to $g_{X Y}=2 \sum_{i=1}^{2}\left(\partial_{1} x_{\varepsilon}^{i}\left(X^{j}\right)\right)\left(\partial_{2} x_{\varepsilon}^{i}\left(X^{j}\right)\right)=: 2 \sum_{i=1}^{2} S_{i}$. Inserting from (10) we have

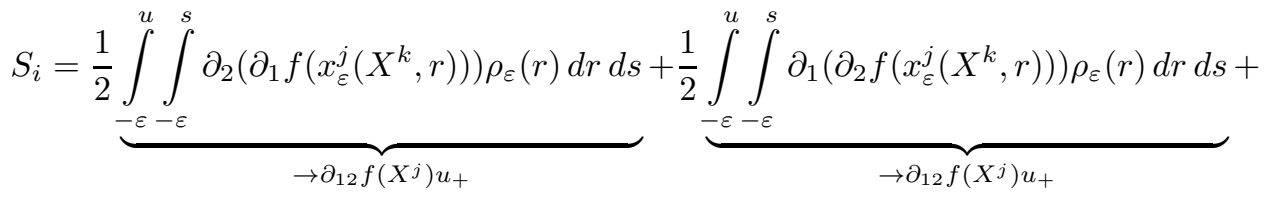

$$
\begin{aligned}
& \underbrace{\frac{1}{4} \underbrace{u}_{-\varepsilon} \int_{-\varepsilon}^{s} \partial_{2}\left(\partial_{i} f\left(x_{\varepsilon}^{j}\left(X^{k}, r\right)\right)\right) \rho_{\varepsilon}(r) d r d s \int_{-\varepsilon}^{u} \int_{-\varepsilon}^{s} \partial_{1}\left(\partial_{i} f\left(x_{\varepsilon}^{j}\left(X^{k}, r\right)\right)\right) \rho_{\varepsilon}(r) d r d s}_{=: D}
\end{aligned}
$$

We claim that

$$
D \rightarrow \partial_{1 i} f\left(X^{j}\right) \partial_{2 i} f\left(X^{j}\right) u_{+}^{2}
$$

To establish this, let $\varphi \in \mathcal{D}$ and consider

$$
\begin{aligned}
& \langle D, \varphi\rangle-\partial_{1 i} f\left(X^{j}\right) \partial_{2 i} f\left(X^{j}\right) \int_{0}^{\infty} u^{2} \varphi(u) d u=\int_{-\varepsilon}^{\infty}\left(\int_{-\varepsilon-\varepsilon}^{u} \int_{-\varepsilon}^{s} \partial_{1}\left(\partial_{i} f\left(x_{\varepsilon}^{j}\left(X^{k}, r\right)\right)\right) \rho_{\varepsilon}(r) d r d s\right) \\
& \left(\int_{-\varepsilon}^{u} \int_{-\varepsilon}^{s} \partial_{2}\left(\partial_{i} f\left(x_{\varepsilon}^{j}\left(X^{k}, r\right)\right)\right) \rho_{\varepsilon}(r) d r d s\right) \varphi(u) d u-\int_{0}^{\infty} \partial_{1 i} f\left(X^{j}\right) \partial_{2 i} f\left(X^{j}\right) u^{2} \varphi(u) d u
\end{aligned}
$$

In order to show that this goes to 0, by a splitting similar to (17) and by the boundedness properties already established it suffices to prove

$$
\begin{aligned}
& \int_{\varepsilon}^{\infty}\left(\int_{\varepsilon}^{u} \int_{-\varepsilon}^{s} \partial_{1}\left(\partial_{i} f\left(x_{\varepsilon}^{j}\left(X^{k}, r\right)\right)\right) \rho_{\varepsilon}(r) d r d s\right)\left(\int_{\varepsilon}^{u} \int_{-\varepsilon}^{s} \partial_{2}\left(\partial_{i} f\left(x_{\varepsilon}^{j}\left(X^{k}, r\right)\right)\right) \rho_{\varepsilon}(r) d r d s\right) \varphi(u) d u- \\
& -\int_{\varepsilon}^{\infty} \partial_{1 i} f\left(X^{j}\right) \partial_{2 i} f\left(X^{j}\right) u^{2} \varphi(u) d u \rightarrow 0,
\end{aligned}
$$

which in turn is a consequence of (19) and (6). Therefore,

$$
g_{X Y} \stackrel{\mathcal{D}^{\prime}}{\rightarrow} \frac{1}{2} u_{+}^{2}\left(\partial_{11} f\left(X^{j}\right) \partial_{21} f\left(X^{j}\right)+\partial_{12} f\left(X^{j}\right) \partial_{22} f\left(X^{j}\right)\right)+2 u_{+} \partial_{12} f\left(X^{j}\right)
$$

From (21), (23), (24) and (26) we obtain the distributional limit of the regularized metric in the form

$$
\begin{aligned}
d s_{\varepsilon}^{2} \stackrel{\mathcal{D}^{\prime}}{\longrightarrow} & -d u d V+\left(1+\frac{1}{2} \partial_{11} f\left(X^{j}\right) u_{+}\right)^{2} d X^{2}+\left(1+\frac{1}{2} \partial_{22} f\left(X^{j}\right) u_{+}\right)^{2} d Y^{2}+ \\
& +\frac{1}{2} \partial_{12} f\left(X^{j}\right) \triangle f\left(X^{j}\right) u_{+}^{2} d X d Y+2 u_{+} \partial_{12} f\left(X^{j}\right) d X d Y+ \\
& +\frac{1}{4}\left(\partial_{12} f\left(X^{j}\right)\right)^{2} u_{+}^{2}\left(d X^{2}+d Y^{2}\right) .
\end{aligned}
$$


Let us sum up the main result of this section in the following diagram

$$
\begin{aligned}
d s^{2}=f\left(x^{i}\right) \delta(u) d u^{2}-d u d v+\sum\left(d x^{i}\right)^{2} & \text { reg. } \\
\qquad ? & T_{\varepsilon}^{2}=f\left(x^{i}\right) \rho_{\varepsilon}(u) d u^{2}-d u d v+\sum\left(d x^{i}\right)^{2} \\
T^{\prime} \text {-limit } & d s_{\varepsilon}^{2}=-d u d V+\left(2 \sum_{i=1}^{2}\left(\dot{x}_{\varepsilon}^{i} \partial_{j} x_{\varepsilon}^{i}\right)-\right. \\
(27)=\lim _{\varepsilon \rightarrow 0} d s_{\varepsilon}^{2} & \left.\partial_{j} v_{\varepsilon}\right) d u d X^{j}+\sum_{i=1}^{2}\left(\partial_{j} x_{\varepsilon}^{i} d X^{j}\right)^{2}
\end{aligned}
$$

The left hand arrow in the above diagram represents the pullback of the distributional metric (5) under the discontinuous coordinate change (see (11))

$$
\begin{aligned}
x^{i} & =X^{i}+\frac{1}{2} \partial_{i} f\left(X^{j}\right) u_{+} \\
v & =V+f\left(X^{j}\right) \theta(u)+\frac{1}{4} \partial_{i} f\left(X^{j}\right) \partial^{i} f\left(X^{j}\right) u_{+} .
\end{aligned}
$$

Note that (28) is the generalization of the Penrose junction conditions (4) to the case of an arbitrary wave profile $f$ (cf. (16) in [13]). Although undefined within Schwartz distribution theory, this transformation can be interpreted consistently as the composition of a regularization procedure, a smooth transformation of the regularized metric and a distributional limit. It is precisely in this sense that the pullback is given by the right hand side of (27) or for short

$$
d s^{2}=-d u d V+\left(\delta_{i j}+\frac{1}{2} u_{+} \partial_{i j} f\right)\left(\delta_{k}^{j}+\frac{1}{2} u_{+} \partial_{k}^{j} f\right) d X^{i} d X^{k}
$$

For the special case of an impulsive plane wave $f(x, y)=x^{2}-y^{2}$ this exactly reduces to (3) and in the general vacuum case it is equivalent to (2) in [11]. Also, we note that (29) appeared first as (17) in [13]. The regularization procedure employed above justifies a posteriori the derivation of (29) by using formal multiplication rules for distributions (cf. the remarks following (4)).

\section{GENERALIZED COORDINATE TRANSFORMATIONS}

In this section we are going to give a precise meaning to the left hand arrow in the above diagram in terms of Colombeau's generalized functions. In order to facilitate the following argument we are going to work in the so called 'simplified' algebra (cf. [10] for a treatment of impulsive pp-waves in the full Colombeau algebra). We note, however, that the considerations to follow retain their validity in the 'full' setting as well. Let $\Omega$ be an open subset of $\mathbb{R}^{n}$. With $\mathcal{E}\left(\Omega ; \mathbb{R}^{n}\right)=\left(\mathcal{C}^{\infty}\left(\Omega ; \mathbb{R}^{n}\right)\right)^{(0, \infty)}$ set

$$
\begin{aligned}
\mathcal{E}_{M}\left(\Omega ; \mathbb{R}^{n}\right):= & \left\{\left(u_{\varepsilon}\right)_{\varepsilon} \in \mathcal{E}\left(\Omega ; \mathbb{R}^{n}\right): \forall K \subset \subset \Omega, \forall m \in \mathbb{N}_{0} \exists p \in \mathbb{N}:\right. \\
& \left.\sup _{x \in K}\left\|D^{(m)} u_{\varepsilon}(x)\right\|=O\left(\varepsilon^{-p}\right)(\varepsilon \rightarrow 0)\right\} \\
\mathcal{N}\left(\Omega ; \mathbb{R}^{n}\right):= & \left\{\left(u_{\varepsilon}\right)_{\varepsilon} \in \mathcal{E}\left(\Omega ; \mathbb{R}^{n}\right): \forall K \subset \subset \Omega, \forall m \in \mathbb{N}_{0} \forall q \in \mathbb{N}:\right. \\
& \left.\sup _{x \in K}\left\|D^{(m)} u_{\varepsilon}(x)\right\|=O\left(\varepsilon^{q}\right)(\varepsilon \rightarrow 0)\right\}
\end{aligned}
$$

where $D^{(m)}$ denotes the $m$-th derivative and $\|\cdot\|$ is any norm on $\mathcal{L}\left(\left(\mathbb{R}^{n}\right)^{m} ; \mathbb{R}^{n}\right)$. Then the simplified Colombeau algebra $\mathcal{G}\left(\Omega ; \mathbb{R}^{n}\right)$ is defined as the differential factor algebra $\mathcal{E}_{M}\left(\Omega ; \mathbb{R}^{n}\right) / \mathcal{N}\left(\Omega ; \mathbb{R}^{n}\right)$ (see [18-20]).

Now let $\Omega, \Omega^{\prime}$ be open subsets of $\mathbb{R}^{4}$ whose $u$-projection contain the interval $[-1,0]$. It is a straightforward consequence of Theorem 1 in [10] and the remarks following (14) that $\left(T_{\varepsilon}\right)_{\varepsilon}$ and $\left(T_{\varepsilon}^{-1}\right)_{\varepsilon}$ (if defined on $\Omega^{\prime}$ ) are elements of $\mathcal{E}_{M}\left(\Omega ; \mathbb{R}^{4}\right)$ and $\mathcal{E}_{M}\left(\Omega^{\prime} ; \mathbb{R}^{4}\right)$, respectively. Let us denote their classes in $\mathcal{G}\left(\Omega ; \mathbb{R}^{4}\right)$ (resp. $\mathcal{G}\left(\Omega^{\prime} ; \mathbb{R}^{4}\right)$ ) by $T$ and $S$. We claim that 
for a suitable choice of $\Omega, \Omega^{\prime}, S$ is the inverse in $\mathcal{G}$ of $T$, which will justify viewing $T$ as a generalized coordinate transformation.

Although composition of Colombeau generalized functions (through componentwise insertion of representatives) is not always well defined, a sufficient condition for this operation to yield a unique Colombeau function was given in [20], 7.3.1 and 7.4.1, to wit: Let $U \in \mathcal{G}\left(\Omega^{\prime} ; \mathbb{R}^{n}\right), V \in \mathcal{G}\left(\Omega ; \mathbb{R}^{n}\right)$, then $U \circ V:=\left[\left(U_{\varepsilon} \circ V_{\varepsilon}\right)_{\varepsilon}\right]$ is a well defined element of $\mathcal{G}\left(\Omega ; \mathbb{R}^{n}\right)$ if there exists a representative $\left(U_{\varepsilon}\right)_{\varepsilon}$ of $U$ such that

$$
\forall K \subset \subset \Omega \exists K^{\prime} \subset \subset \Omega^{\prime} \exists \eta>0 \text { s.t. } U_{\varepsilon}(K) \subseteq K^{\prime} \forall \varepsilon<\eta .
$$

It follows immediately from the remarks following (14) that $S$ satisfies (30). Concerning $T$, suppose that $(u, v, x, y)$ varies in some $K \subset \subset \Omega$ and set $\left(u, V_{\varepsilon}, X_{\varepsilon}, Y_{\varepsilon}\right)=T_{\varepsilon}(u, v, x, y)$. Then

$$
x^{i}=x_{\varepsilon}^{i}\left(X_{\varepsilon}^{j}, u\right)=X_{\varepsilon}^{i}+\frac{1}{2} \int_{-\varepsilon}^{u} \int_{-\varepsilon}^{s} \partial_{i} f\left(x_{\varepsilon}^{j}\left(X_{\varepsilon}^{k}, r\right)\right) \rho_{\varepsilon}(r) d r d s .
$$

Since the left hand side and the argument of $\partial_{i} f$ in this equation are bounded by assumption, $X_{\varepsilon}^{i}$ remains in some compact region $L$ in $\mathbb{R}^{2}$. We already know that on any such set $L$ (times some compact $u$-interval) $\dot{x}_{\varepsilon}^{i}$ is uniformly bounded. Inserting this into

$$
\left.V_{\varepsilon}=v_{\varepsilon}\left(V_{\varepsilon}, X_{\varepsilon}^{i}, u\right)-\int_{-\varepsilon}^{u} f\left(x_{\varepsilon}^{j}\left(X_{\varepsilon}^{k}, s\right)\right) \rho_{\varepsilon}(s) d s-\int_{-\varepsilon}^{u} \int_{-\varepsilon}^{s} \partial_{i} f\left(x_{\varepsilon}^{j}\left(X_{\varepsilon}^{k}, r\right)\right) \dot{x}_{\varepsilon}^{i}\left(X_{\varepsilon}^{k}, r\right)\right) \rho_{\varepsilon}(r) d r d s
$$

also establishes the desired boundedness property for $V_{\varepsilon}$. We conclude that $S$ is indeed the $\mathcal{G}$-inverse of $T$ (for suitable regions $\Omega, \Omega^{\prime}$ ). Therefore the left hand arrow in the above diagram, i.e. the (generalized) Penrose junction conditions (28) turn out to be the macroscopic aspect (or 'distributional shadow') of the (pullback under the) generalized coordinate transformation $T$.

\section{CONCLUSIONS}

In this work we have studied the discontinuous coordinate transformation relating the distributional with the continuous form of the impulsive pp-wave metric. It is indeed possible to interpret this change of coordinates as the (distributional) limit of a family of smooth transformations which we obtained by a general regularization proceedure. We emphasize the fact that our results are completely regularization independent even within the maximal class of regularizations of the Dirac- $\delta$, hence "natural". Note also that we did not have to impose Einstein's equations throughout our analysis, hence our results apply to all pp-waves, i.e. even to the non-vacuum case.

Physically speaking our regularization approach consists in viewing the impulsive wave as a limiting case of a sandwich wave with an arbitrarily regularized wave profile. From this point of view the two forms of the impulsive metric arise as the (distributional) limits of the sandwich wave in different coordinate systems. It is a well known fact [22] that given a one-parameter family of spacetimes one may obtain different limits by introducing a suitable (parameter dependent) change of coordinates. This effect is mainly caused by a different identification of points in the manifold. More precisely, the transformation in the limit sends finite points to infinity, hence is unbounded. However, in our case the limit of the transformation, although discontinuous, remains bounded and the mentioned ambiguities do not arise. This is in fact not surprising since our change of coordinates is adjusted to the geometry of the spacetime which (in the sense of geodesic completeness) is nonsingular, allowing only for a finite jump of the geodesics.

Finally, the family of smoothened transformations constitutes a generalized coordinate transformation in the sense of Colombeau, whose macroscopic (i.e. distributional) aspect is precisely given by the (generalized) Penrose junction conditions (28).

These results put the formal calculations (using certain ad-hoc multiplication rules for distributions) on solid ground and set up a framework which allows to also mathematically identify the two "physically equivalent" descriptions of impulsive waves.

\section{ACKNOWLEDGEMENT}

It's a pleasure for us to thank all the members of the DIANA research group (http://diana.mat.univie.ac.at/ diana) for providing such an inspiring working environment. We also thank H. Urbantke for his comments on the draft version 
of this paper. M. Kunzinger was supported by Research Grant P10472-MAT of the Austrian Science Foundation. R. Steinbauer was supported by Austrian Academy of Science, Ph.D. programme, grant \#338 and by Research Grant P12023-MAT of the Austrian Science Foundation.

[1] D. Kramer, H. Stephani, M. MacCallum, E. Herlt, Exact Solutions of Einstein's Field equations, (Cambridge University Press, Cambridge, 1980).

[2] R. Penrose, "The geometry of impulsive gravitational waves," in General Relativity, Papers in Honour of J. L. Synge, edited by L. O'Raifeartaigh (Clarendon Press, Oxford, 1972), pp. 101-115.

[3] P. C. Aichelburg, R. U. Sexl, "On the gravitational field of a massless particle," J. Gen. Rel. Grav. 2, 303-312 (1971).

[4] C. O. Loustó, N. Sánchez, "The ultrarelativistic limit of the Kerr-Newman geometry and particle scattering at the Planck scale," Phys. Lett. B 232, 462-466 (1989).

[5] H. Balasin, H. Nachbagauer, "The ultrarelativistic Kerr-geometry and its energy-momentum tensor," Class. Quantum Grav. 12, 707-713 (1995).

[6] J. Podolsky, J. B. Griffiths, "Boosted static multipole particles as sources of impulsive gravitational waves," Phys. Rev. D. 58, 124024 (1998).

[7] E. Verlinde, H. Verlinde, "High-energy scattering in Quantum Gravity," Class. Quant. Grav. 10, Suppl. 175-184 (1993).

[8] S. de Haro, "Planckian Scattering and Black Holes," JHEP 9810, 023 (1998).

[9] R. Steinbauer, "Geodesics and geodesic deviation for impulsive gravitational waves," J. Math. Phys. 39, $2201-2212$ (1998).

[10] M. Kunzinger, R. Steinbauer, "A rigorous solution concept for geodesic and geodesic deviation equations in impusive gravitational waves," J. Math. Phys. 40, 1479-1489 (1999).

[11] J. Podolsky, K. Vesely, "Continuous coordinates for all impulsive pp - waves," Phys.Lett. A241, 145-147 (1998).

[12] J. Podolsky, K. Vesely, "New examples of sandwich gravitational waves and their impulsive limit," Czech. J. Phys. 48, 871, (1998).

[13] P. C. Aichelburg, H. Balasin, "Generalized symmetries of impulsive gravitational waves," Class. Quant. Grav. 14, A31-A41, (1997).

[14] C.J.S. Clarke, T. Dray, "Junction conditions for null hypersurfaces," Class. Quant. Grav. 4, 265-275 (1987).

[15] R. Steinbauer, "On the geometry of impulsive gravitational waves," gr-qc/9809054, to appear in Proceedings of the VIII Romanian Conference on General Relativity, edited by I. Cotaescu, D. Vulcanov, (Mirton Publishing House, Timisoara, 1998).

[16] O. Hájek, Bull. AMS 12, 272-279 (1985).

[17] R. Steinbauer, "The ultrarelativistic Reissner-Nordstrøm field in the Colombeau algebra," J. Math. Phys. 38, 1614-1622 (1997).

[18] M. Oberguggenberger, "Multiplication of Distributions and Applications to Partial Differential Equations," (Longman Scientific and Technical, New York, 1992).

[19] J. F. Colombeau, "Elementary Introduction to New Generalized Functions," (North Holland, Amsterdam, 1985).

[20] J. Aragona, H. A. Biagioni, "Intrinsic definition of the Colombeau algebra of generalized functions," Analysis Mathematica, 17, 75 - 132, (1991).

[21] D. Gale, H. Nikaido, "The Jacobian matrix and global univalence of mappings," Math. Ann., 159, 81-93 (1965).

[22] R. Geroch, "Limits of spacetimes," Comm. Math. Phys. 13, 180-193, (1969). 